\title{
On the Influence of Acoustic Reflection on the Inertial Cavitation Dose in a Franz Diffusion Cell
}

\author{
Jeremy Robertson ${ }^{1}$ and Sid Becker ${ }^{1}$ \\ ${ }^{1}$ Mechanical Engineering Department, University of Canterbury, Christchurch, New Zealand
}

Address correspondence to: Sid Becker, Mechanical Engineering Department, Private Bag 4800, Christchurch 8041, New Zealand. E-mail: sid.becker@canterbury.ac.nz Phone: $+6433692155$ 
Abstract-The exposure of the skin to low-frequency $(20-100 \mathrm{kHz})$ ultrasound is a wellestablished method for increasing its permeability to drugs. The mechanism behind this permeability increase has been shown to be inertial cavitation within the coupling fluid. This study investigated the influence of acoustic reflections on the inertial cavitation dose during low-frequency $(20 \mathrm{kHz})$ exposure in an in vitro skin sonoporation setup. This investigation was conducted using a passive cavitation detector that monitored the broadband noise emission within a modified Franz diffusion cell. Two versions of this diffusion cell were employed. One version had acoustic conditions that were similar to a standard Franz diffusion cell surrounded by air, while the second was designed to greatly reduce the acoustic reflection by submerging the diffusion cell in a water bath. The temperature of the coupling fluid in both setups was controlled using a novel thermoelectric cooling system. At an ultrasound intensity of 13.6 W/cm ${ }^{2}$ the median inertial cavitation dose when the acoustic reflections were suppressed was found to be only about $15 \%$ lower than when reflections were not suppressed.

Index Terms-Inertial Cavitation, Franz Diffusion Cell, Sonoporation, Passive Cavitation Detection. 


\section{Introduction}

Ultrasound enhanced transdermal drug delivery involves the exposure of the skin to an ultrasound field in order to increase the skin's permeability to topical drugs. The mechanism responsible for this permeability increase is believed to be inertial cavitation (Tang, et al. 2002, Tezel, et al. 2002, Tezel, et al. 2001) and is characterized by the violent collapse of gaseous cavities within the coupling fluid. This is believed to result in the disruption of the lipid bilayer structure of the stratum corneum (Azagury, et al. 2014).

In vitro experimental studies that investigate the effects of ultrasound on skin permeability employ Franz diffusion cells (Merino, et al. 2003, Mitragotri, et al. 2000, Smith, et al. 2003, Terahara, et al. 2002, Tezel, et al. 2001). These studies have considered the influences of parameters such as frequency (Tezel, et al. 2002), exposure time (Terahara, et al. 2002), duty cycle (Herwadkar, et al. 2012), acoustic intensity (whether it be spatial peak temporal peak, or spatial peak temporal average etc.) (Tezel, et al. 2001), distance from the transducer to the skin (Terahara, et al. 2002), and chemical composition of the coupling fluid (Lavon, et al. 2005). However, little attention has been paid to the potential influence of acoustic reflection in skin sonoporation studies. This was noted by Smith (2007), who stressed the importance of reporting the echoic conditions of the transdermal insonation apparatus.

Previous studies of the insonation of cells in cell culture plates have shown that acoustic reflection from the walls of the cell culture plates may strongly influence the inertial cavitation activity. For example, Kinoshita and Hynynen (2007) illustrated that sonoporation could be doubled by facilitating the presence of a standing wave. Similarly, Jelenc, et al. (2012) showed that by suppressing the acoustic reflection using an acoustically absorbent lining, the maximum 
reflected pressure (at the cell suspension) was reduced by $81 \%$. This motivates the question that is central to the present study: at ultrasound intensities relevant to skin sonoporation, do the acoustic reflections that occur within a Franz diffusion cell significantly influence the inertial cavitation dose?

While, to the authors' knowledge, there are no published studies that explicitly investigate the influence of reflection in in vitro skin sonoporation setups, some researchers have employed experimental methods intended to suppress these reflections. For example, in their highfrequency in vivo study on transdermal transport through rat skin, Park, et al. (2012) positioned a thermocouple outside of the direct beam of their transducer in order to avoid strong reflection. Mitragotri, et al. (1995) inserted a $1 \mathrm{~mm}$ thick Teflon sheet opposite the transducer in a highfrequency horizontal diffusion cell setup in an attempt to reduce multiple ultrasound reflections. In their high-intensity focussed ultrasound (HIFU), in vitro study on transdermal transport through pig skin, Helga, et al. (2015) inserted a polyurethane absorber opposite the transducer in order to avoid reflections of the HIFU beam.

The presence of inertial cavitation has previously been shown to be observable by passive cavitation detection (PCD) (Farny, et al. 2010, Hallow, et al. 2006, Helga, et al. 2015, Liu, et al. 1998). In a PCD setup, a hydrophone is placed directly into the coupling fluid and confocally aligned with the ultrasound beam in order to measure the ultrasound pressure fluctuations that are radiated by the cavitation bubbles during ultrasound application. The voltage vs. time data that this hydrophone records during ultrasound application is then filtered to isolate the broadband noise. The RMS value of the broadband noise is indicative of the magnitude of the inertial cavitation within the ultrasound field (Hallow, et al. 2006). Cell sonoporation studies, such as those conducted by Chen, et al. (2003) and Hallow, et al. (2006), have shown that it is 
the cavitation dose, as opposed to the magnitude of broadband noise emission, that best correlates with cavitation-induced enhancement of a bioeffect such as hemolysis or intracellular uptake. Because cavitation dose includes a temporal component, any changes in cavitation activity over the course of ultrasound exposure will alter the cavitation dose value.

While PCDs have been frequently employed in studies involving the insonation of media other than skin (Coussios and Roy 2008, Farny, et al. 2010, Liu, et al. 1998, Yao-Sheng, et al. 2010), they have rarely been used in low-frequency in vitro skin insonation studies. This is probably due to the geometric constraints within the traditional Franz diffusion cell which make it difficult to fit both a transducer and a hydrophone in the coupling fluid. In the study by Tang, et al. (2002) a hydrophone was fixed to the outer surface of the bottom of the receiver chamber. This allowed for the PCD data to be recorded during skin insonation but did not directly capture the ultrasound field signal within the coupling fluid. Tezel and Mitragotri (2003) were able to directly monitor the ultrasound field in the coupling fluid, but could not take measurements during skin insonation as the hydrophone was positioned where the skin sample would usually be positioned. An in vitro skin insonation apparatus that allows for a hydrophone to be positioned in the coupling fluid during skin insonation has not yet been reported. In part, the motivation of the current work was to develop an experimental apparatus that allows the PCD data to be gathered from the Franz diffusion cell during the application of the ultrasound.

The application of ultrasound at intensities required for significant inertial cavitation has been shown to result in considerable heating of the coupling fluid due to absorption (O'Brien Jr 2007, Tezel, et al. 2001). This heating is an important issue as extended exposure to temperatures as low as $40{ }^{\circ} \mathrm{C}$ has been shown to increase skin permeability (Park, et al. 2008) even though stratum corneum lipid fluidisation does not occur until around $65^{\circ} \mathrm{C}$ (Golden, et 
al. 1986, Golden, et al. 1987). Two approaches to addressing this heating have been reported in the literature. Some studies replaced the coupling fluid within the donor cell during the application of the ultrasound field in order to limit the maximum temperature of the skin (Polat, et al. 2012, Tang, et al. 2002, Terahara, et al. 2002). Alternatively, duty cycles have been used in which the total exposure time was administered at regularly spaced intervals allowing the coupling fluid to cool off in the time between applications (Lavon, et al. 2005, Merino, et al. 2003, Mitragotri, et al. 2000, Paliwal, et al. 2006, Terahara, et al. 2002). The replacement of the coupling fluid and the use of duty cycles were both able to maintain the coupling fluid temperature below some specified maximum value. However, as cavitation activity is temperature dependent (Brabec and Mornstein 2007), a temperature control method that facilitates a constant coupling fluid temperature during the continuous application of high intensity ultrasound would be more appropriate. Such a method is presented in the current study.

In this study we introduce a modified Franz diffusion cell in which the transducer and the hydrophone were able to be simultaneously positioned in the coupling fluid. This allowed the cavitation dose to be calculated from data taken directly from the actual donor cell during insonation. The diffusion cell setup also employed a novel temperature control method to mitigate the coupling fluid temperature increases brought on by continuous ultrasound application. We used this setup to investigate whether the acoustic reflections that occur within a Franz diffusion cell significantly influence the inertial cavitation dose at an intensity relevant to skin sonoporation. 


\section{Materials and Methods}

\section{Modified Franz Diffusion Cell}

The modified Franz diffusion cell consisted of a donor chamber that was fixed to a receiver chamber with a specially designed acoustically transmissive clamp. The donor chamber had an inner diameter of $15 \mathrm{~mm}$, an outer diameter of $19 \mathrm{~mm}$ and an aperture diameter of $9 \mathrm{~mm}$. The total volume of the donor chamber was $6.7 \mathrm{~mL}$. The receiver chamber also had a $9 \mathrm{~mm}$ aperture, and a volume of $3.4 \mathrm{~mL}$. The donor, receiver, and clamp geometries are presented in Fig. 1.

The donor chamber was 3D printed from VeroClear (VeroClear-RGD810, Stratasys Ltd, Minnesota, USA). The receiver chamber was turned from a solid polypropylene rod (Polystone, Dotmar EPP Pty Ltd, Christchurch, New Zealand) on a CNC lathe (Top-Turn CNC 406, Jashico Machine Manufacture Co. LTD, Taichung, Taiwan). A piece of carbon fibre tubing with an inner diameter of $3.5 \mathrm{~mm}$ (Carbon Fibre Tube Pultruded, MAKERshop, Auckland, New Zealand) was glued to the polypropylene receiver with Loctite 401 to form the sampling arm. In order to enable coupling fluid circulation and temperature control, two pieces of carbon fibre tubing with inner diameters of $2 \mathrm{~mm}$ were glued to the donor chamber to form ports (Carbon Fibre Tube Pultruded, MAKERshop, Auckland, New Zealand). The clamp was 3D printed from Acrylonitrile Butadiene Styrene (ABS) (ABS P430, Stratasys Ltd, Minnesota, USA).

A $16 \mathrm{~mm}$ diameter, $1 \mathrm{~mm}$ thick piece of polypropylene was inserted between the donor and receiver chambers. This acted as a skin phantom. Like skin, polypropylene has a similar acoustic impedance to water. Therefore, the phantom provided the solid surface necessary for 
a-symmetrical bubble collapse without diminishing the intensity of the ultrasound waves that were transmitted through to the receiver fluid. The surface roughness of a material has been shown to influence the cavitation activity that arises when that material is insonated (Nour, et al. 2007). Therefore, the inertial cavitation activity that arises from the insonation of polypropylene may be different to that which arises from the insonation of skin under the same ultrasound conditions. The focus of this study was to assess the influence of the diffusion cell's acoustic conditions on the inertial cavitation dose. Therefore, the phantom was used as it provided a consistent target surface condition for inertial cavitation activity. Two polystyrene gaskets (Permagear Inc, Pennsylvania, USA) were placed between the donor and receiver chambers on each side of the phantom.

\section{Coupling Fluid Temperature Measurement and Control}

The temperature of the coupling fluid was measured with a thermocouple (Wire Type K Thermocouple, Jaycar Electronics Pty Ltd, Auckland, New Zealand). The relative position of the thermocouple can be seen in Fig. 2. The readings from the thermocouple were passed to a NI-9211 DAQ module (National Instruments Ltd., Texas, USA) then on to a PC for processing in LabVIEW (National Instruments Ltd., Texas, USA). The thermocouple sampling frequency was $1 \mathrm{~Hz}$. The readings of the thermocouple were found to be independent of thermocouple position in the donor chamber.

In order to maintain a constant coupling fluid temperature, the fluid was circulated out of the donor chamber, through a custom built temperature controller and back into the donor chamber at a flow rate of $0.14 \mathrm{~L} / \mathrm{min}$. This circuit is depicted in Fig. 3. A peristaltic pump (6 V Peristaltic Pump, Amazon, Washington, USA) was used to achieve this flow rate. The components in the cooling circuit were linked by $2.6 \mathrm{~mm}$ inner diameter silicone tubing (Silicone Tubing Clear 
Grade, Rubbermark Industries Ltd., Auckland, New Zealand). This tubing could be easily removed from the donor chamber ports to allow for replacement of fluid between each experiment.

The temperature controller consisted of six $12 \mathrm{~V}$ thermoelectric Peltier plates (Peltier $60 \mathrm{~W}$, TecParts NZ Ltd., Tauranga, New Zealand) arranged around a block of aluminium. As the coupling fluid passed through a series of connected holes drilled in the aluminium block it was cooled. The cooling rate was controlled by the power supply to the Peltier plates. The hot sides of the Peltier plates were connected to water cooled fins. These fins were constructed from square cross-sectioned aluminium tubing that had a side length of $40 \mathrm{~mm}$, a thickness of $3 \mathrm{~mm}$, and an overall length of $300 \mathrm{~mm}$. A thermally conductive paste (Heatsink Compound, Jaycar Electronics Pty Ltd, Auckland, New Zealand) was applied to both sides of each Peltier plate to ensure effective heat transfer. The coupling fluid temperature was maintained at $15 \pm 1{ }^{\circ} \mathrm{C}$.

\section{Ultrasound Field Generation}

A $20 \mathrm{kHz}$ ultrasound field was generated using an ultrasonic processor (VC 505, Sonics and Materials Inc., Connecticut, USA) with a $13 \mathrm{~mm}$ diameter replaceable tip. The intensity of the ultrasound field produced by this system could be varied by altering the tip displacement. All of the experiments in this study used continuous exposure to the ultrasound field (100\% duty cycle) for an application time of 10 minutes. Terahara, et al. (2002) showed that the effectiveness of low-frequency skin sonoporation increases with decreasing distance between the transducer face and the target region. A distance of $5 \mathrm{~mm}$ was used for the experiments in the present study as this was the smallest distance that still allowed for the hydrophone to be confocal with the transducer. 
The near-field to far-field transition distance, $L$, was determined from the relation (Sprawls 1995)

$$
L=\frac{D^{2}}{4 \lambda}
$$

where $D$ is the diameter of the active face of the transducer $(13 \mathrm{~mm})$ and $\lambda$ is the wavelength $(73 \mathrm{~mm})$, calculated with the speed of sound in water (at $15{ }^{\circ} \mathrm{C}$ ) of $1466 \mathrm{~m} / \mathrm{s}$. For these parameters, the value of $L$ was calculated to be $0.58 \mathrm{~mm}$. Therefore, at the distance of $5 \mathrm{~mm}$, the skin phantom was well outside the near-field, which is common practice in lowfrequency skin sonoporation.

\section{Determination of Ultrasound Intensity}

The ultrasound intensity was determined using the calorimetric method (Merino, et al. 2003, Mitragotri, et al. 2000, Mitragotri, et al. 2000, Morimoto, et al. 2005, Mutoh, et al. 2003, Terahara, et al. 2002). In the determination of the ultrasound intensity values, the ultrasound transducer was positioned in a $200 \mathrm{~mL}$ beaker of water embedded in a thermally insulating block of polystyrene. The transducer was then operated at a variety of tip displacement amplitudes in order to vary the resulting ultrasound intensity. The intensity resulting from operation at each of the tip displacement amplitudes $(I)$ was calculated from

$$
I=\left(\frac{m C_{P}}{A}\right) \frac{\Delta T}{\Delta t}
$$

where $m$ is the mass of water in the beaker $(0.3 \mathrm{~kg}), C_{P}$ is the specific heat of water $(4.187 \mathrm{~kJ} / \mathrm{kgK}), A$ is the active transducer face area $\left(1.3 \mathrm{~cm}^{2}\right)$, and $\Delta T$ is the change in 
temperature that occurred during the 3 minutes $(\Delta t)$ of ultrasound exposure. Each of these intensity measurements was run in triplicate. An intensity of $13.7 \mathrm{~W} / \mathrm{m}^{2}$, which was achieved with a tip displacement setting of $20 \%$ on the VC 505 ultrasonic processor, was used for all experiments in this study. This was a spatial average temporal average intensity as in the study by (Mitragotri, et al. 2000). This intensity level was chosen as both Terahara, et al. (2002) and Mitragotri, et al. (2000) achieved considerable skin conductivity increases with intensities between 5 and $15 \mathrm{~W} / \mathrm{m}^{2}$ when using the same diameter transducer tip that was used in this study.

\section{Passive Cavitation Detector Alignment}

The acoustic measurements were taken with a $2 \mathrm{~mm}$ needle hydrophone $(2.0 \mathrm{~mm}$ Needle Hydrophone, Precision Acoustics Ltd, Dorchester, Dorset, UK), which had a sensitivity of $-236.4 \mathrm{dBre} 1 \mathrm{~V} / \mu \mathrm{Pa}$ at the transducer driving frequency of $20 \mathrm{kHz}$. The manufacturer coated the tip of this hydrophone in silicone to reduce the potential of cavitation damage. A depiction of the relative position of the hydrophone in the diffusion cell is presented in Fig. 2. The hydrophone was pointed directly at the centre of the skin phantom so that it was confocal with the transducer. The tip of the hydrophone was $20 \mathrm{~mm}$ from the centre of the skin phantom further ensuring that the hydrophone tip was not damaged by cavitation. Note that the hydrophone was held in its fixed position using an aluminium sleeve (Fig. 4) that was fixed in place relative to the diffusion cell. In this way the hydrophone could be easily inserted and removed between experiments. Before each experiment, the hydrophone alignment was checked (with the transducer in place) by ensuring that a mark on the centre of the skin phantom was visible when looking through the centre of the empty sleeve. 
The voltage signal measured by the hydrophone was recorded with an oscilloscope (TDS 2014B, Tektronix, Beaverton, OR, USA) connected via USB to a PC running SignalExpress (NI LabVIEW SignalExpress, National Instruments, Texas, USA). The oscilloscope collected 2500 data points at a sampling rate of $1 \mathrm{MHz}$ and then transferred these to the PC. The oscilloscope then collected another 2500 data points after a delay of around 1 to 2 seconds. This continued for the 10 minute duration of insonation.

\section{Determination of Inertial Cavitation Dose Using Broadband Noise Emission}

Each set of 2500 data points was filtered so that the broadband noise (indicative of inertial cavitation) could be isolated from the harmonic and sub-harmonic peaks in the frequency spectrum. A digital bandpass filter isolated a band of noise between 92.5 and $97.5 \mathrm{kHz}$. When choosing the frequency range of this band, two factors were considered: that no harmonics or sub-harmonics should be included in the chosen filter band, as these are influenced by noninertial cavitation (Yao-Sheng, et al. 2010); and that the frequency band should be low enough to allow for a minimum of 10 data points per period to be sampled by the oscilloscope in order to ensure that the hydrophone signal was accurately represented.

The infinite impulse response bandpass filter was produced using MATLAB's 'designfilt' function (MATLAB, MathWorks, Natick, MA, USA). A filter order of 20 was used as this was found to be the minimum order capable of providing the necessary reduction of the $5^{\text {th }}$ harmonic at $100 \mathrm{kHz}$ and the subharmonic at $90 \mathrm{kHz}$.

Once each set of the 2500 data points had been filtered, the RMS value was calculated for the $2.5 \mathrm{~ms}$ time period associated with those data points. This RMS value then represented one noise amplitude data point. The inertial cavitation dose was calculated by numerically 
integrating the noise amplitude over the 10 minutes of ultrasound application in each experiment. Therefore, the inertial cavitation dose had units of V-s as in the cell sonoporation studies by Chen, et al. (2003) and Hallow, et al. (2006). This numerical integration was evaluated using the MATLAB 'trapz' function.

\section{Acoustic Reflection Experiments}

Two sets of experiments were devised in order to investigate the influence of acoustic reflections on inertial cavitation dose. In one set of experiments, the diffusion cell was surrounded by air to replicate the echoic conditions within a traditional Franz diffusion cell. In the second set of experiments, acoustic reflection was greatly reduced by submerging the diffusion cell in a water bath so that the water line was $5 \mathrm{~mm}$ below the top of the donor chamber (Fig. 5). The water bath was $794 \mathrm{~mm}$ long, $396 \mathrm{~mm}$ wide, and $362 \mathrm{~mm}$ high. The walls of the water bath were made from $12 \mathrm{~mm}$ thick acrylic sheets. To further reduce reflection, the interior walls of one half of the water bath (the half containing the diffusion cell) were lined with acoustically absorbent panels (SA-J35, Hangzhou Applied Acoustic Institute, Hangzhou, Zhejiang, China). These panels have been shown to decrease low-frequency ultrasound reflection by $20 \mathrm{~dB}$ (Jelenc, et al. 2012). The panels were $300 \mathrm{~mm}$ wide, $300 \mathrm{~mm}$ long, and had a thickness that varied from $10 \mathrm{~mm}$ to $35 \mathrm{~mm}$ due to their wedged front surface. The unlined half of the water bath was used for the coupling fluid temperature control system.

The proportion of ultrasound intensity that was reflected $(R)$ at the interface of the diffusion cell and surrounding medium in each of the two setups can be approximated by the relation (Cracknell 1980): 


$$
R=\left(\frac{Z_{2}-Z_{1}}{Z_{2}+Z_{1}}\right)^{2}
$$

where $Z_{1}$ is the acoustic impedance of the material that the acoustic wave is propagating from (the coupling fluid) and $Z_{2}$ is the acoustic impedance of the material the acoustic wave is propagating into (the surrounding medium). Given the acoustic impedance values for polypropylene (2.4 MRayl), water (1.5 MRayl), and air (400 Rayl), the R values are calculated as 0.99 for the setup surrounded by air and 0.05 for the setup submerged in water (note that the acoustic impedance of the 3D printed VeroClear donor chamber was approximated to be equal to that of polypropylene). These values indicate that the proportion of ultrasound intensity that was reflected back into the coupling fluid was approximately 20 times greater for the cell surrounded by air.

Seven repetitions of the experiments were conducted for each of the two setups $(n=7)$. Each experiment consisted of 10 minutes of ultrasound exposure. This application time was chosen to reflect those of published low-frequency skin sonoporation studies (Sarheed and Abdul Rasool 2011, Terahara, et al. 2002, Tezel, et al. 2001). The coupling fluid (deionised water) was replaced between each experimental repetition. The same phantom was used in each experimental repetition as no cavitation damage was observed on its surface. 


\section{Results}

The amplitude of the broadband noise has been reported to be an effective indicator of the presence of inertial cavitation in insonation setups (Farny, et al. 2010, Hallow, et al. 2006, Helga, et al. 2015, Liu, et al. 1998). In order to illustrate the relationship between inertial cavitation and broadband noise in the current setup, the standard configuration (with air as the surrounding medium) was used to capture the PCD frequency spectra under three different insonation conditions. First, the frequency spectrum in the absence of any applied ultrasound $\left(0 \mathrm{~W} / \mathrm{cm}^{2}\right)$ was captured in order to illustrate the noise floor (Fig. 6a). The noise floor in the frequency band of interest ( 92.5 to $97.5 \mathrm{kHz}$ ) had a magnitude of around $-250 \mathrm{~dB}$ (relative to $1 \mathrm{~V})$. Next, two frequency spectra resulting from insonation at $13.7 \mathrm{~W} / \mathrm{m}^{2}$ were captured. For one of these, the coupling fluid was deionised water, while for the other, the coupling fluid was castor oil. Castor oil has a similar acoustic impedance to water but a much higher viscosity (Polat, et al. 2011), therefore, it subdues inertial cavitation (Tang, et al. 2002) without removing the harmonic and sub-harmonic peaks in the frequency spectrum. With this in mind, frequency spectra corresponding to insonation without inertial cavitation suppression and insonation with inertial cavitation suppression are presented in Fig. $6 \mathrm{~b}$ and Fig. $6 \mathrm{c}$ respectively. When inertial cavitation was not suppressed, the broadband noise emission magnitude in the frequency band of interest was around $-160 \mathrm{~dB}$, far greater than the noise floor of $-250 \mathrm{~dB}$. When inertial cavitation was suppressed using castor oil as the coupling fluid, the broadband noise emission magnitude was approximately $-220 \mathrm{~dB}$. Although this magnitude is still somewhat higher than the noise floor, it is significantly lower than the deionised water case. This indicates that broadband noise emission, within this frequency range, was a likely indicator of inertial cavitation activity in the present diffusion cell setup. 
Under certain conditions, the application of ultrasound in a diffusion cell has been shown to result in an increase in coupling fluid temperature due to absorption (O'Brien Jr 2007): the fluid medium absorbs a portion of the ultrasound energy, converting it into heat. In order to maintain constant temperatures during insonation in this study, the coupling fluid was circulated through a thermoelectric cooling device during the application of ultrasound at an intensity of $13.7 \mathrm{~W} / \mathrm{cm}^{2}$. The effectiveness of the cooling device was apparent when the coupling fluid temperature without cooling was compared to the coupling fluid temperature with cooling over 10 minutes of insonation (Fig. 7). In the absence of temperature control, the coupling fluid temperature increased to $50{ }^{\circ} \mathrm{C}$ within $110 \mathrm{~s}$. With cooling, the temperature was able to be maintained at $15 \pm 1^{\circ} \mathrm{C}$.

Both temperature and broadband noise emission were monitored over 10 minutes of insonation in a diffusion cell surrounded by air and in a diffusion cell submerged in water. The broadband noise data for one of the seven repetitions that was collected when the cell was surrounded by air is presented in Fig. 8a. The broadband noise data for one of the seven repetitions that was collected when the cell was submerged in water is presented in Fig. 8b. Transient temperature data is not shown here, but the coupling fluid temperature was always maintained at $15 \pm 1{ }^{\circ} \mathrm{C}$.

In the study by Bull, et al. (2013), a simple method was used to determine whether broadband noise emission exceeded a threshold level representative of statistically significant inertial cavitation activity. The ' $100 \%$ occurrence threshold' methodology dictates that all filtered broadband noise readings must be above a minimum baseline level in order for the inertial cavitation threshold to be surpassed. This baseline noise level, $N_{B L}$, was determined from the relation, 


$$
N_{B L}=\mu+4 \sigma
$$

where $\mu$ is the mean value of the noise floor and $\sigma$ is the standard deviation of the noise floor. The baseline noise level for the present setup is depicted by the dashed lines at the bottom of Fig. 8a and Fig. 8b. All of the broadband noise emission data in Fig. 8a and Fig. 8b was well above this threshold. This indicates that the level of the broadband noise observed during each of the seven repetitions in each of the two diffusion cells was due to inertial cavitation activity as opposed to other potential sources of noise such as electromagnetic interference.

The broadband noise emission data was converted to a cavitation dose value for each of the seven repetitions conducted in each of the two diffusion cell setups. Figure 9 displays boxplots of the cavitation dose data. The central horizontal line in each of the boxes represents the median value, the cross represents the mean value, the top and bottom horizontal line of each box represents the upper and lower quartiles, and the whiskers represent the maximum and minimum values in each set.

There was a difference between the median and mean dose values for the two setups. When the surrounding medium was air, the median and mean values were $1590 \mathrm{mV}$-s and $1709 \mathrm{mV}$-s respectively. When the surrounding medium was water, the median and mean values were $1385 \mathrm{mV}$-s and $1433 \mathrm{mV}$-s respectively. Therefore, the suppression of acoustic reflection resulted in a $13 \%$ decrease in the median inertial cavitation dose and a $16 \%$ decrease in the mean inertial cavitation dose. 
There was also a difference in the degree of variability for the two cases. When the surrounding medium was air, the standard deviation was $255 \mathrm{mV}$-s. When the surrounding medium was water, the standard deviation was $149 \mathrm{mV}$-s. This difference in variability was anticipated because an increase in the mean inertial cavitation dose has been reported to result in an increase in standard deviation (Chen, et al. 2003).

\section{Discussion}

The results of this study indicate that, at an ultrasound intensity of $13.6 \mathrm{~W} / \mathrm{cm}^{2}$, the inertial cavitation dose was only slightly influenced by the acoustic reflections in the diffusion cell setup. However, it is unclear from the results in Fig. 9 whether the acoustic reflections within the diffusion cell would influence the results of a skin sonoporation study by a statistically significant amount especially considering that skin sonoporation studies often contain a high degree of variability (Mitragotri, et al. 2000, Schoellhammer, et al. 2012, Terahara, et al. 2002, Tezel, et al. 2001).

The present study was in part motivated by the results of cell sonoporation studies which indicated that acoustic reflections can have a strong influence on inertial cavitation activity. We developed an experiment to investigate whether acoustic reflections in experimental conditions associated with skin sonoporation would also influence inertial cavitation. The influence of acoustic reflections found in the present study was minor in comparison to that found in a study which investigated the influence of acoustic reflections in a cell sonoporation setup. Kinoshita and Hynynen (2007) found that cell sonoporation was halved when acoustic reflections were suppressed. One explanation for the much smaller influence of reflection in 
the present study compared to the cell sonoporation study may be related to the different setup geometries used in the experiments. The setup geometry affects the intensity of the reflected ultrasound waves in the target region, due to the distance that the reflected waves must travel before returning to the target region from the point of reflection. The target region in the setup of Kinoshita and Hynynen (2007) was the cell culture plate, while the target region in the present setup was the coupling fluid near the surface of the phantom. The main acoustically reflective interface in the cell culture plate was the top of the cell culture plate (which was within the target region), whereas the main acoustically reflective interface in the Franz diffusion cell was the bottom of the receiver chamber which was outside the target region (by approximately $40 \mathrm{~mm}$ in this case). As the reflected wave fronts had to travel a shorter distance in the study by Kinoshita and Hynynen (2007) than they did in the present study, they caused a greater amount of acoustic interference, and therefore, lead to a greater increase in the peak rarefactional pressure in the target region.

\section{Conclusions}

A modified Franz diffusion cell was developed so that a PCD hydrophone could be positioned confocally with the transducer during ultrasound application. This allowed for PCD data to be captured during the application of ultrasound. This enabled the inertial cavitation dose to be employed as a means for quantifying inertial cavitation behaviour. This setup was used to investigate the effect of acoustic reflection on the inertial cavitation dose during lowfrequency insonation at an intensity applicable to skin sonoporation $\left(13.6 \mathrm{~W} / \mathrm{cm}^{2}\right)$. When acoustic reflection was suppressed, the mean inertial cavitation dose was only about $10 \%$ lower than when the reflection was not supressed. This result suggests that, at an intensity and 
frequency relevant to skin sonoporation, the acoustic reflections within a low-frequency Franz diffusion cell skin sonoporation setup do not significantly influence inertial cavitation during insonation.

The coupling fluid circulation and cooling system enabled the continuous application of ultrasound (at intensity relevant to skin sonoporation) for a period of 10 minutes without any notable increase in the temperature of the coupling fluid. In order to demonstrate that this method is a viable alternative to duty cycles and periodic coupling fluid replacement, future work should be conducted to quantify the effect that the coupling fluid circulation has on inertial cavitation activity and sonoporation efficacy.

The present study represents an initial inquiry into the influence of acoustic reflections in skin sonoporation setups. While the intensity and frequency used in this study are applicable to skin sonoporation (Mitragotri, et al. 2000, Terahara, et al. 2002), further investigation of the influence of acoustic reflections in Franz diffusion cells should consider a range of frequencies, intensities and target materials if a complete understanding of the underlying physics is to be obtained. 


\section{Acknowledgment}

The authors greatly appreciate the help of Dr Nataša Pavšelj and Dr Barbara Zorec, Faculty of Electrical Engineering, University of Ljubljana, and Dr Jure Jelenc, Iskra Medical d.o.o., Slovenia. Also, Julian Phillips and Rob McGregor, University of Canterbury. This work was supported in part by the Royal Society of New Zealand's Marsden Fund. 


\section{References}

Azagury A, Khoury L, Enden G, Kost J. Ultrasound mediated transdermal drug delivery. Adv Drug Deliv Rev 2014; 72:127-43.

Brabec K, Mornstein V. Detection of ultrasonic cavitation based on low-frequency analysis of acoustic signal. Cent Eur J Biol 2007; 2:213-21.

Bull V, Civale J, Rivens I, ter Haar G. A comparison of acoustic cavitation detection thresholds measured with piezo-electric and fiber-optic hydrophone sensors. Ultrasound Med Biol 2013; 39:240621.

Chen W-S, Brayman AA, Matula TJ, Crum LA. Inertial cavitation dose and hemolysis produced in vitro with or without Optison. Ultrasound Med Biol 2003; 29:725-37.

Coussios CC, Roy RA. Applications of Acoustics and Cavitation to Noninvasive Therapy and Drug Delivery. Annu Rev Fluid Mech 2008; 40:395-420.

Cracknell AP. Ultrasonics. London: Wykeham Publication, 1980.

Farny CH, Holt RG, Roy RA. The Correlation Between Bubble-Enhanced HIFU Heating and Cavitation Power. IEEE Trans Biomed Eng 2010; 57:175-84.

Golden GM, Guzek DB, Harris RR, McKie JE, Potts RO. Lipid Thermotropic Transitions in Human Stratum Corneum. Journal of Investigative Dermatology 1986; 86:255-59.

Golden GM, Guzek DB, Kennedy AE, McKie JE, Potts RO. Stratum corneum lipid phase transitions and water barrier properties. Biochemistry 1987; 26:2382-88.

Hallow DM, Mahajan AD, McCutchen TE, Prausnitz MR. Measurement and correlation of acoustic cavitation with cellular bioeffects. Ultrasound Med Biol 2006; 32:1111-22.

Helga F, Sunali B, Miklós G, Constantin CC. Cavitation-enhanced delivery of insulin in agar and porcine models of human skin. Phys Med Biol 2015; 60:2421.

Herwadkar A, Sachdeva V, Taylor LF, Silver H, Banga AK. Low frequency sonophoresis mediated transdermal and intradermal delivery of ketoprofen. Int J Pharm 2012; 423:289-96.

Jelenc J, Jelenc J, Miklavcic D, Lebar AM. Low-Frequency Sonoporation in vitro: Experimental System Evaluation. Strojniški vestnik 2012; 58:319-26.

Kinoshita M, Hynynen K. Key factors that affect sonoporation efficiency in in vitro settings: The importance of standing wave in sonoporation. Biochem Biophys Res Commun 2007; 359:86065.

Lavon I, Grossman N, Kost J. The nature of ultrasound-SLS synergism during enhanced transdermal transport. J Control Release 2005; 107:484-94.

Liu J, Lewis T, Prausnitz M. Non-Invasive Assessment and Control of Ultrasound-Mediated Membrane Permeabilization. Pharm Res 1998; 15:918-24.

Merino G, Kalia YN, Delgado-Charro MB, Potts RO, Guy RH. Frequency and thermal effects on the enhancement of transdermal transport by sonophoresis. J Control Release 2003; 88:85-94.

Mitragotri S, Edwards DA, Blankschtein D, Langer R. A mechanistic study of ultrasonically-enhanced transdermal drug delivery. J Pharm Sci 1995; 84:697-706.

Mitragotri S, Farrell J, Tang H, Terahara T, Kost J, Langer R. Determination of threshold energy dose for ultrasound-induced transdermal drug transport. J Control Release 2000; 63:41-52.

Mitragotri S, Ray D, Farrell J, Tang H, Yu B, Kost J, Blankschtein D, Langer R. Synergistic effect of lowfrequency ultrasound and sodium lauryl sulfate on transdermal transport. J Pharm Sci 2000; 89:892-900.

Morimoto Y, Mutoh M, Ueda H, Fang L, Hirayama K, Atobe M, Kobayashi D. Elucidation of the transport pathway in hairless rat skin enhanced by low-frequency sonophoresis based on the solutewater transport relationship and confocal microscopy. J Control Release 2005; 103:587-97. 
Mutoh M, Ueda H, Nakamura Y, Hirayama K, Atobe M, Kobayashi D, Morimoto Y. Characterization of transdermal solute transport induced by low-frequency ultrasound in the hairless rat skin. J Control Release 2003; 92:137-46.

Nour WMN, Dulias U, Schneider J, Gahr KHZ. The effect of surface finish and cavitating liquid on the cavitation erosion of alumina and silicon carbide ceramics, 2007.

O’Brien Jr WD. Ultrasound-biophysics mechanisms. Prog Biophys Mol Bio 2007; 93:212-55.

Paliwal S, Menon GK, Mitragotri S. Low-Frequency Sonophoresis: Ultrastructural Basis for Stratum Corneum Permeability Assessed Using Quantum Dots. J Invest Dermatol 2006; 126:1095-101.

Park D, Ryu H, Kim HS, Kim Y-s, Choi K-S. Sonophoresis using ultrasound contrast agents for transdermal drug delivery: an in vivo experimental study. Ultrasound Med Biol 2012; 38:64250.

Park J-H, Lee J-W, Kim Y-C, Prausnitz MR. The effect of heat on skin permeability. Int J Pharm 2008; 359:94-103.

Polat BE, Deen WM, Langer R, Blankschtein D. A physical mechanism to explain the delivery of chemical penetration enhancers into skin during transdermal sonophoresis - Insight into the observed synergism. J Control Release 2012; 158:250-60.

Polat BE, Hart D, Langer R, Blankschtein D. Ultrasound-mediated transdermal drug delivery: Mechanisms, scope, and emerging trends. J Control Release 2011; 152:330-48.

Sarheed O, Abdul Rasool BK. Development of an Optimised Application Protocol For Sonophoretic Transdermal Delivery of a Model Hydrophilic Drug. Open Biomed Eng J 2011; 5:14-24.

Schoellhammer CM, Polat BE, Mendenhall J, Maa R, Jones B, Hart DP, Langer R, Blankschtein D. Rapid skin permeabilization by the simultaneous application of dual-frequency, high-intensity ultrasound. J Control Release 2012; 163:154-60.

Smith N, Lee S, Maione E, Roy RB, McElligott S. Ultrasound-mediated transdermal transport of insulin in vitro through human skin using novel transducer designs. Ultrasound Med Biol 2003; 29:311-17.

Smith NB. Perspectives on transdermal ultrasound mediated drug delivery. Int J Nanomedicine 2007; 2:585-94.

Sprawls P. The Physical Principles of Medical Imaging. Wisconsin: Medical Physics Publishing, 1995.

Tang H, Wang CCJ, Blankschtein D, Langer R. An Investigation of the Role of Cavitation in LowFrequency Ultrasound-Mediated Transdermal Drug Transport. Pharm Res 2002; 19:1160-69.

Terahara T, Mitragotri S, Kost J, Langer R. Dependence of low-frequency sonophoresis on ultrasound parameters; distance of the horn and intensity. Int J Pharm 2002; 235:35-42.

Tezel A, Mitragotri S. Interactions of Inertial Cavitation Bubbles with Stratum Corneum Lipid Bilayers during Low-Frequency Sonophoresis. Biophys J 2003; 85:3502-12.

Tezel A, Sens A, Mitragotri S. Investigations of the role of cavitation in low-frequency sonophoresis using acoustic spectroscopy. J Pharm Sci 2002; 91:444-53.

Tezel A, Sens A, Tuchscherer J, Mitragotri S. Frequency Dependence of Sonophoresis. Pharm Res 2001; 18:1694-700.

Yao-Sheng T, Choi JJ, Konofagou EE. Identifying the Inertial Cavitation Pressure Threshold and Skull Effects in a Vessel Phantom Using Focused Ultrasound and Microbubbles. AIP Conf Prec 2010; 1215:186-89. 
Figure Captions
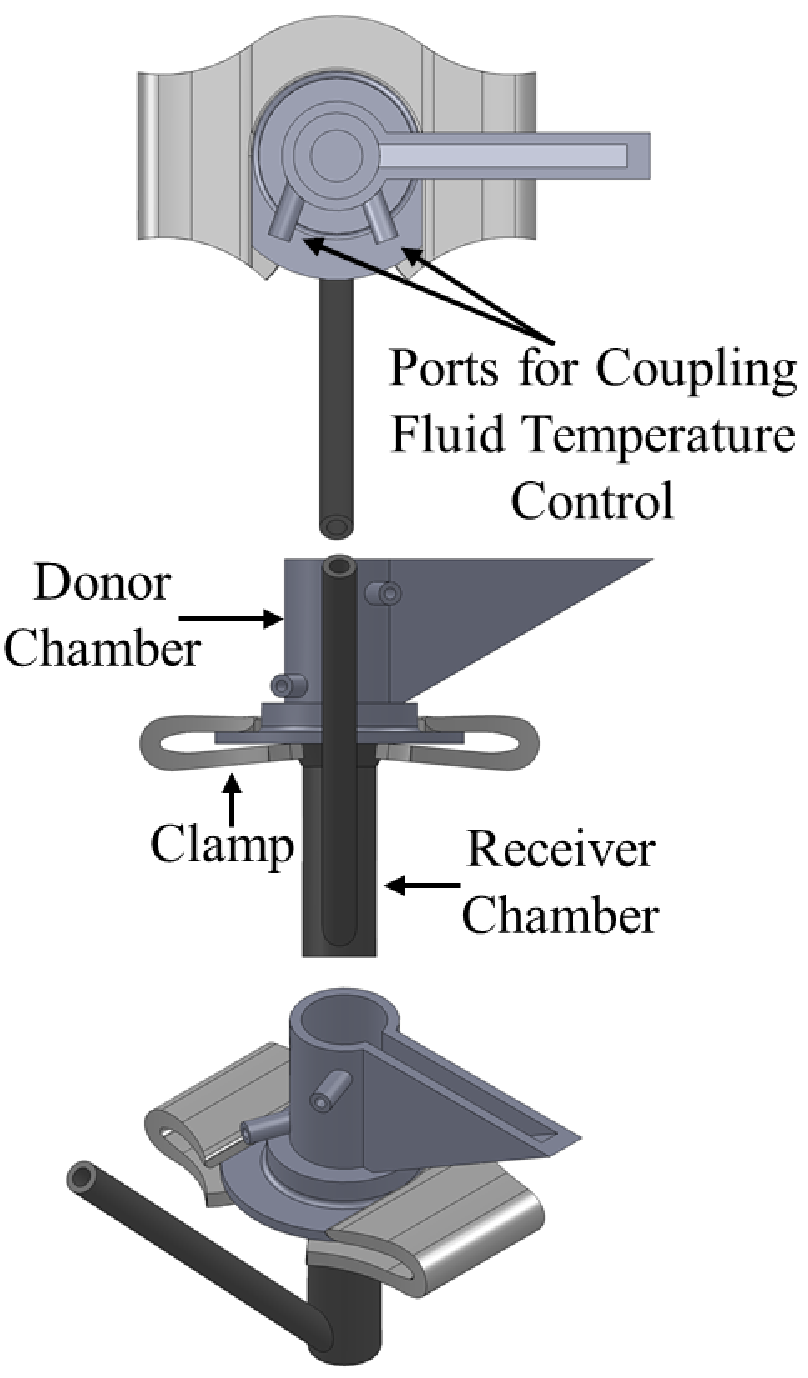

Fig. 1 Modified diffusion cell geometry (top) plan view, (middle) front view, and (bottom) isometric view 


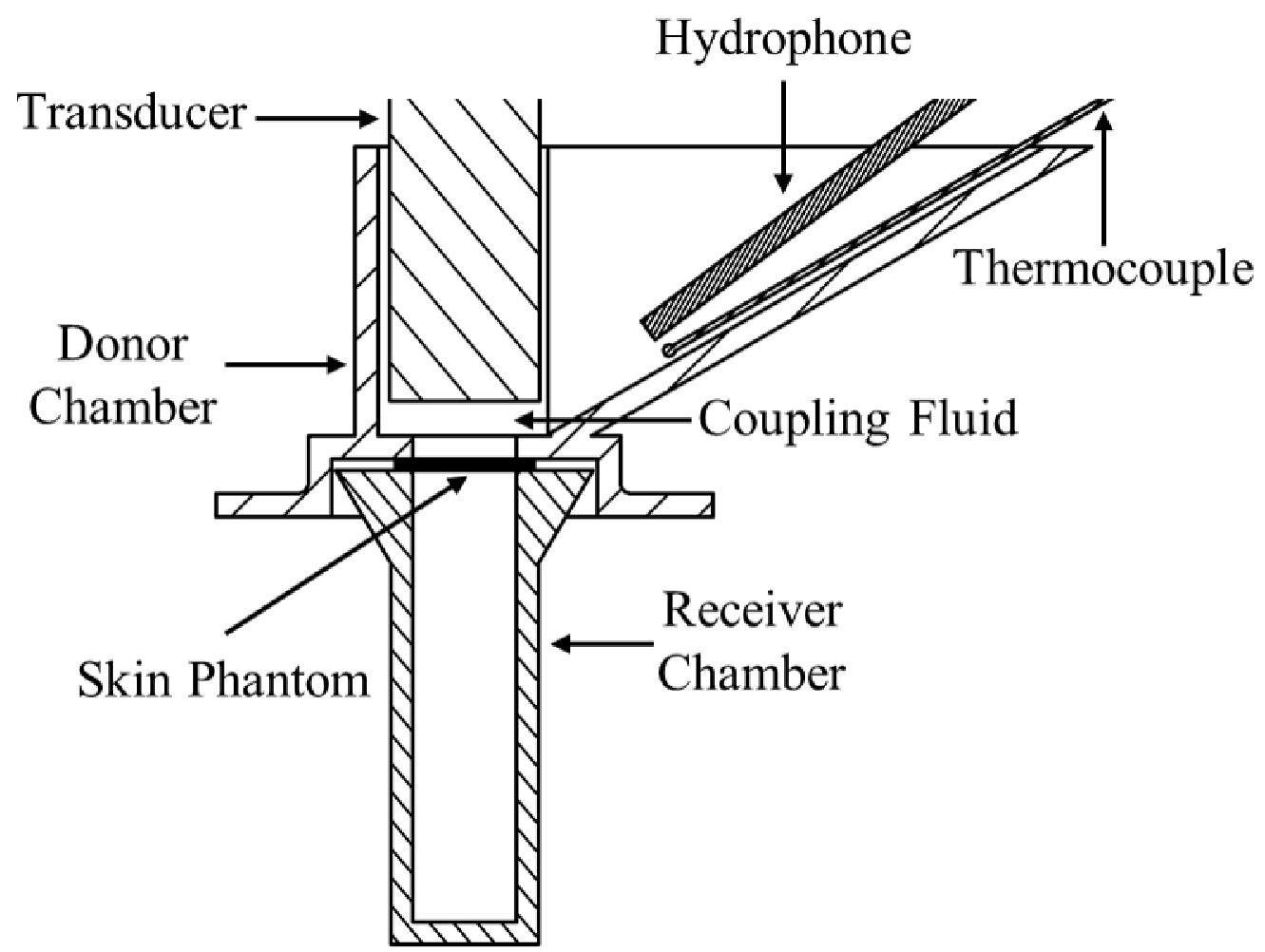

Fig. 2 Cross section of the diffusion cell showing the positioning of the transducer, thermocouple, and hydrophone 


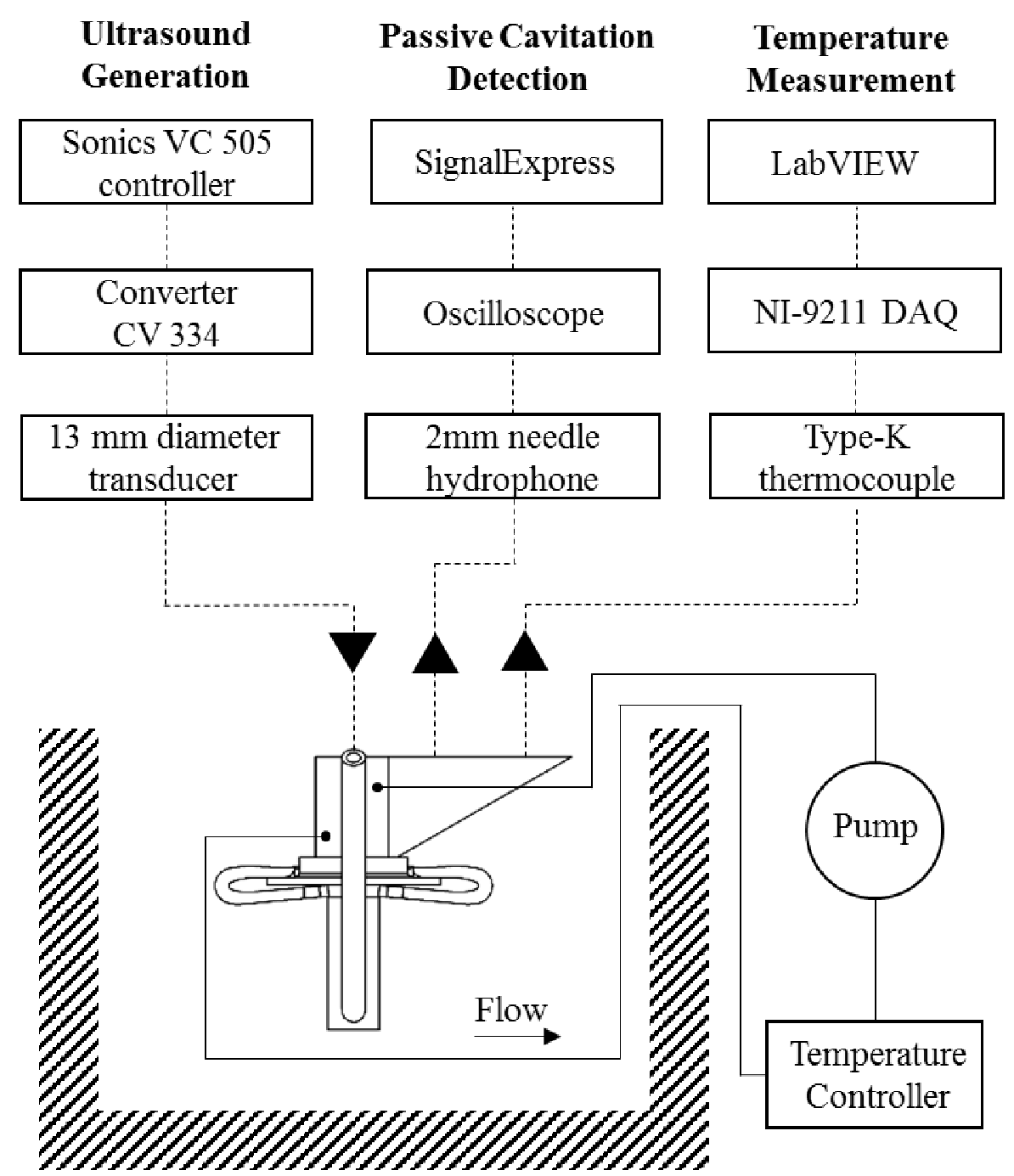

Acoustically Absorbent Panels

Fig. 3 System schematic. The solid lines represent the coupling fluid circuit. The dashed lines represent the signal inputs and outputs to and from the instruments in the coupling fluid. 


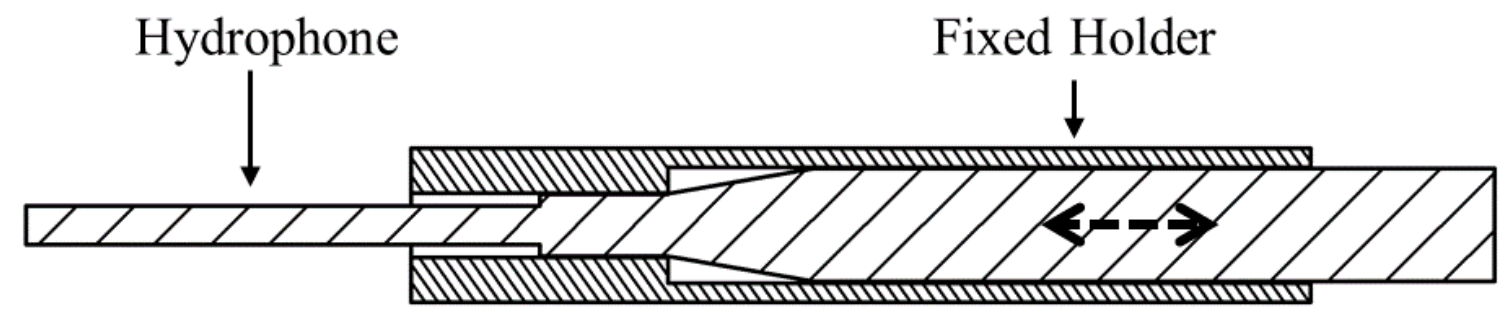

Fig. 4 Cross section of the hydrophone sleeve showing the direction of hydrophone movement during removal and reinsertion 


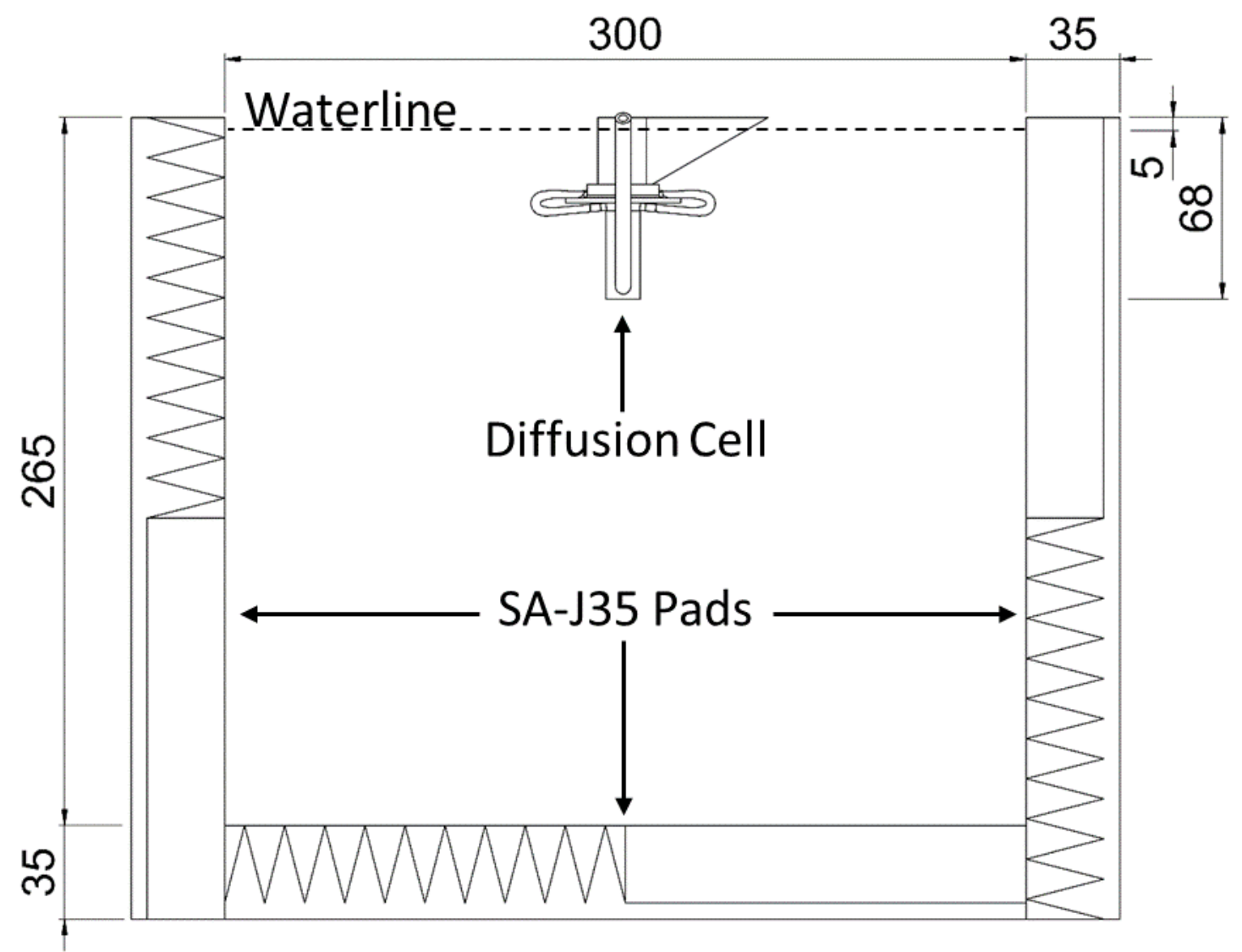

Fig. 5 Section view of the diffusion cell in the water bath which was lined with acoustically absorbent SA-J35 pads. The overall height of the diffusion cell is shown along with the waterline which was $5 \mathrm{~mm}$ below the top of the donor chamber. 

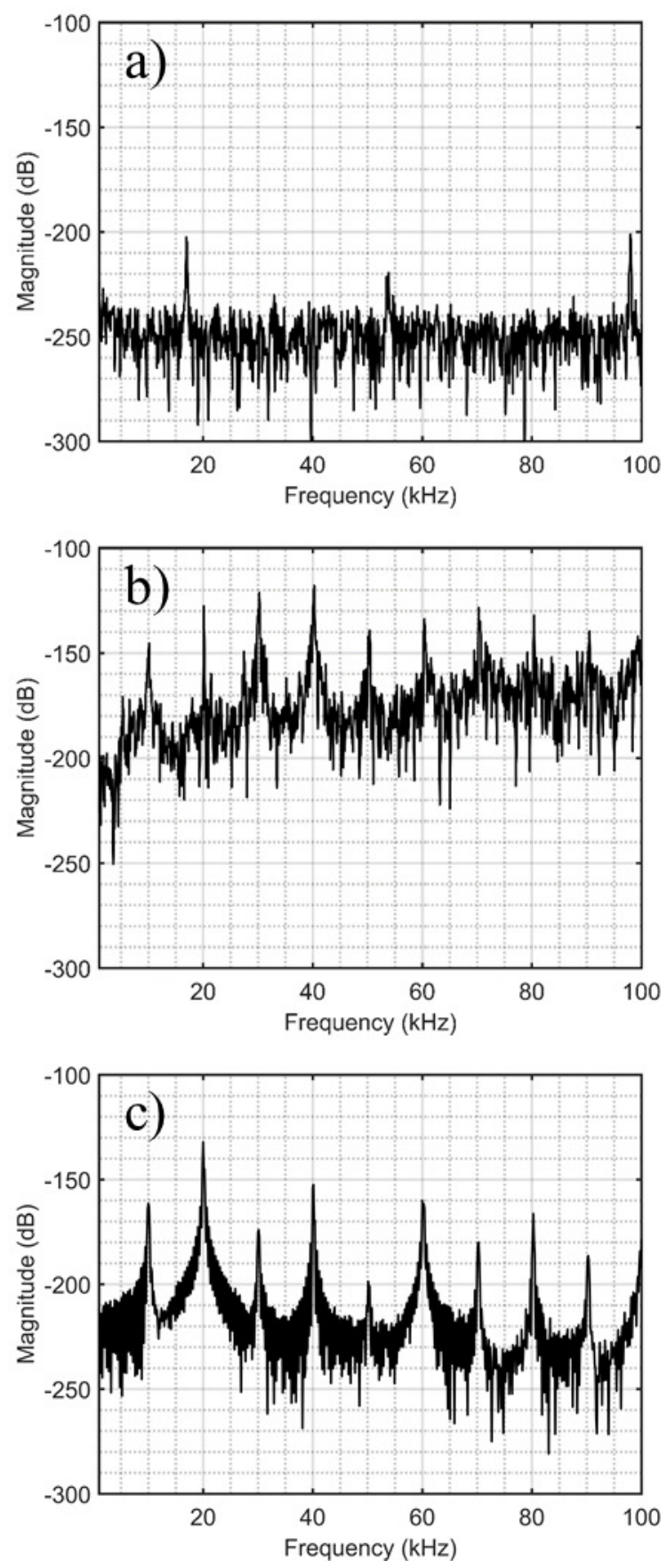

Fig. 6 PCD frequency spectra in (a) the absence of ultrasound, $(b)$ the presence of ultrasound with deionised water as the coupling fluid, and (c) the presence of ultrasound with castor oil as the coupling fluid 


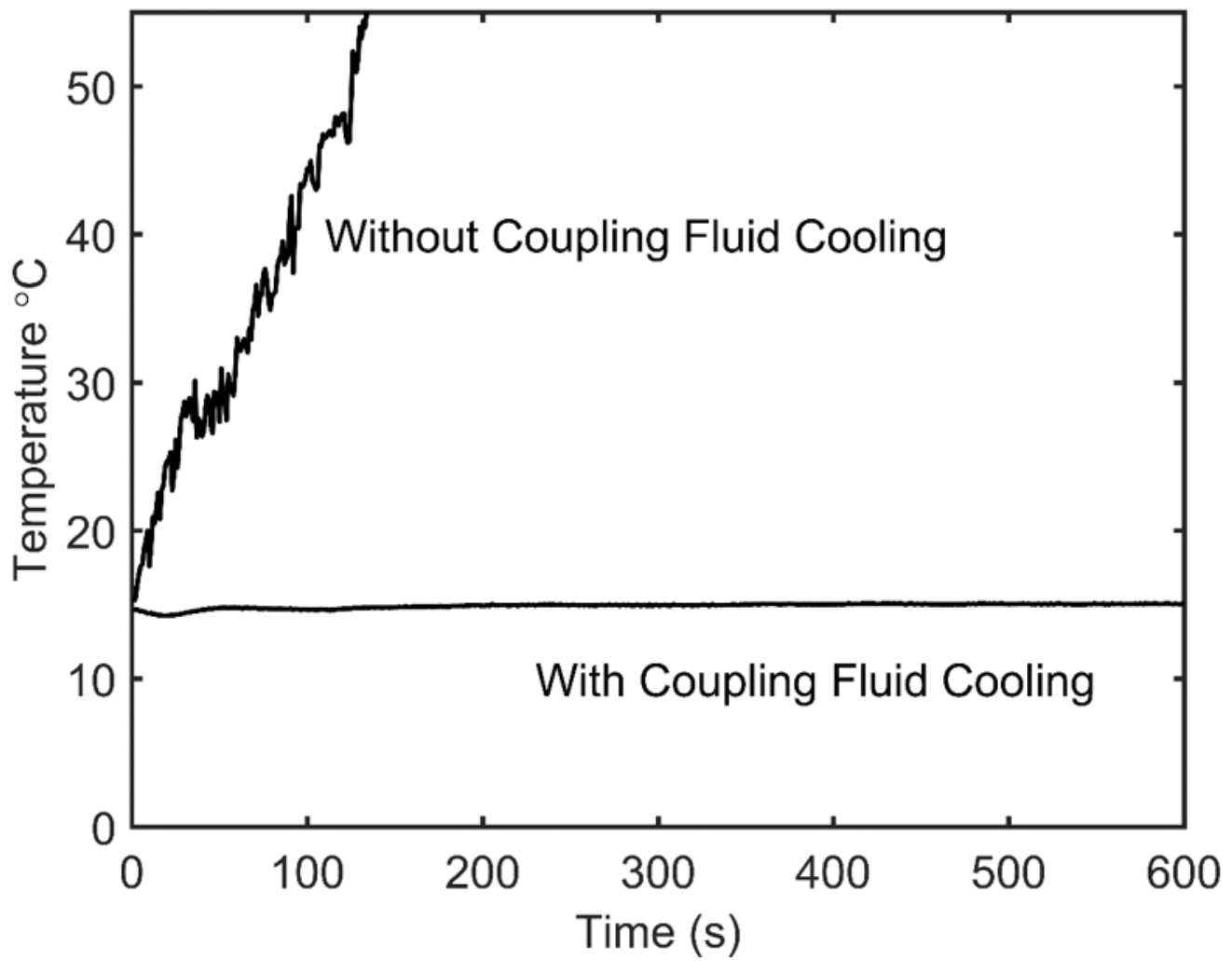

Fig. 7 The transient behaviour of the coupling fluid temperature with and without coupling fluid cooling during insonation at an intensity of $13.7 \mathrm{~W} / \mathrm{cm}^{2}$ 

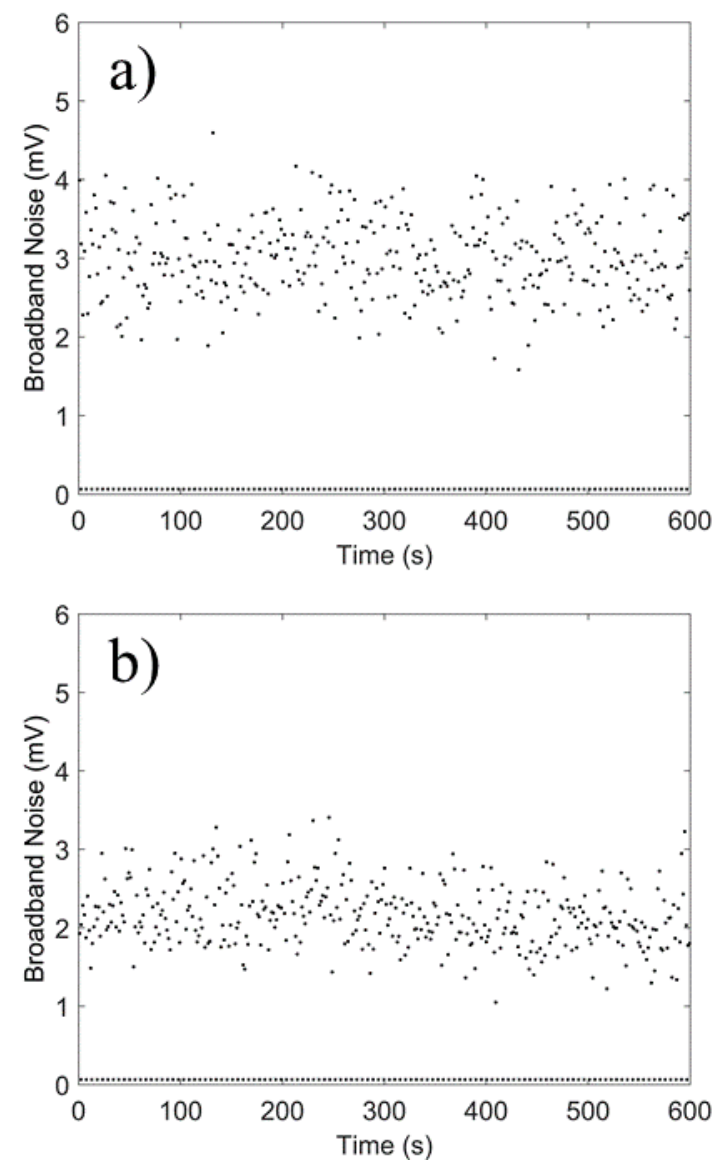

Fig. 8 Examples of the broadband noise data captured over 10 minutes of continuous ultrasound application at an intensity of $13.7 \mathrm{~W} / \mathrm{cm}^{2}$ with the coupling fluid maintained at $15 \pm l^{\circ} \mathrm{C}$ in (a) a diffusion cell surrounded by air, and (b) a diffusion cell submerged in water.

The dashed lines at the very bottom indicate the baseline noise level. 


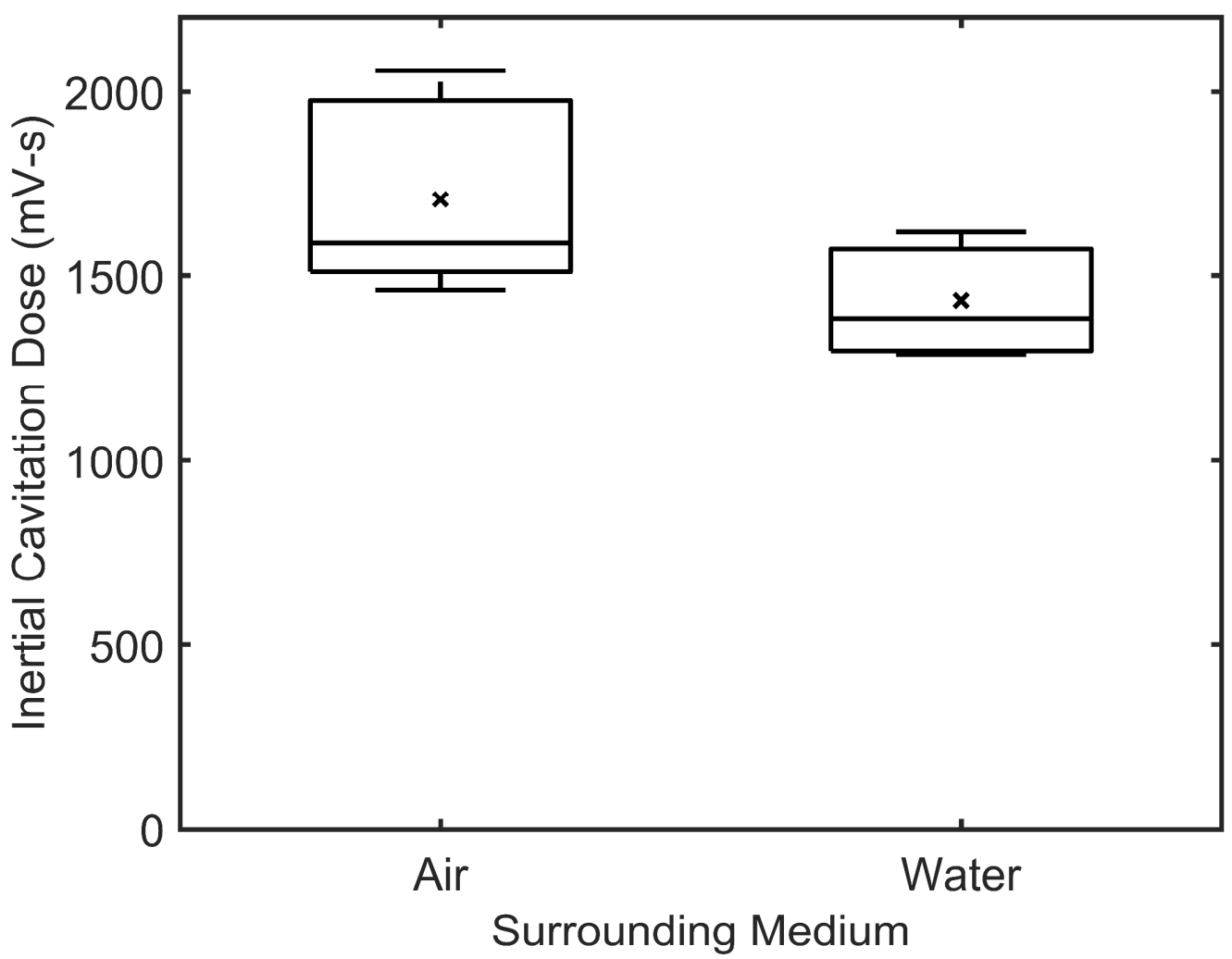

Fig. 9 Inertial cavitation dose calculated for the diffusion cell setup surrounded by air and the diffusion cell setup submerged in water $(n=7)$. Ultrasound was applied at an intensity of $13.7 \mathrm{~W} / \mathrm{cm}^{2}$ continuously for 10 minutes. The coupling fluid was maintained at $15 \pm 1{ }^{\circ} \mathrm{C}$. 